\title{
Linguistic encoding in short-term memory as a function of stimulus type
}

\author{
HARLEY HAMILTON \\ Atlanta Area School for the Deaf, Clarkston, Georgia \\ and \\ THOMAS G. HOLZMAN \\ Georgia State University, Atlanta, Georgia
}

\begin{abstract}
In this study, we investigated bases for encoding linguistic stimuli in short-term memory. Past research has provided evidence for both phonological (sound-based) and cherological (sign-based) encoding, the former typically found with hearing subjects and the latter with deaf users of sign language. In the present experiment, encoding capabilities were delineated from encoding preferences, using 58 subjects comprising six groups differing in hearing ability and linguistic experience. Phonologically related, cherologically related, and control lists were presented orally, manually, or through both modalities simultaneously. Recall performance indicated that individuals encode flexibly, the code actually used being biased by incoming stimulus characteristics. Subjects with both sign and speech experience recalled simultaneous presentations better than ones presented orally or manually alone, which reveals the occurrence of enhanced encoding as a function of linguistic experience. Total linguistic experience appeared to determine recall accuracy following different types of encoding, rather than determining the encoding basis used.
\end{abstract}

In this study, we investigated the required and preferred bases for encoding linguistic material in short-term memory (STM). Conflicting evidence has been presented on this issue, stemming in part from the relatively homogeneous sensory capabilities and linguistic experiences of the subjects in most previous studies.

Most investigations of linguistic encoding in STM have been conducted with hearing subjects. Much of the early research had posited that linguistic stimuli are phonologically encoded in STM, regardless of presentation mode (e.g., Atkinson \& Shiffrin, 1968; Baddeley, 1966, 1976; Conrad, 1962, 1964, 1972; Craik, 1970; Crowder, 1976; Crowder \& Morton, 1969; Hintzman, 1967; Hurley \& Kamil, 1976; Neisser, 1967; Sperling, 1960, 1963; Sperling \& Speelman, 1970). Besides the strong evidence for a phonological basis for encoding linguistic stimuli in STM, research has also indicated that other encoding bases are possible. For instance, some studies have suggested that semantic encoding in STM can occur (Shulman, 1970, 1971, 1972; Wetherick, 1975; Wickens, 1972; Zhang \& Simon, 1985). However, Baddeley (1972) argued that this semantic encoding is due to retrieval rules stored in longterm memory and found no reason to abandon the sim-

This article is based on the first author's doctoral dissertation submitted to Georgia State University. The first author is grateful to Douglas J. Stanwyck and W. L. Ballard who, together with the second author, supervised this research as members of his dissertation committee. Many thanks also to Frank Caccamise of the National Technical Institute for the Deaf for his assistance in recruiting subjects, and to Ursula Bellugi for her helpful comments on earlier versions of this text. Correspondence may be addressed to Harley Hamilton, Atlanta Area School for the Deaf, 890 North Indian Creek Dr., Clarkston, GA 30021 pler generalization that phonological encoding dominates STM. Other studies have suggested that there are also STM capabilities for encoding linguistic information visually (Agrawal \& Sarkaria, 1981; Camden, Motley, \& Baars, 1982; Janata, Joelson, Joss, \& Herrmann, 1978; Zhang \& Simon, 1985). Again, however, these authors contend that phonological encoding is dominant even though visual coding is possible. Still other research on STM encoding has found evidence for simultaneous acoustical/phonological encoding and visual encoding of letters, and has thus suggested that dual encoding of linguistic material can occur (Hakoda \& Nakamizo, 1975; Heyer \& Barrett, 1971).

Studies using deaf subjects have found that printed verbal stimuli could be encoded on the basis of their shapes (Conrad, 1971; Locke \& Locke, 1971; Wallace \& Corballis, 1973) or on the basis of finger-spelling codes (Hanson, Liberman, \& Shankweiler, 1984; Locke \& Locke, 1971; Wallace \& Corballis, 1973). Cherological encoding (i.e., encoding based on the formational parameters of sign) by native deaf signers has also been shown to occur in several studies (Bellugi, Klima, \& Siple, 1975; Bellugi \& Siple, 1974; Hirsh-Pasek \& Treiman, 1982; Krakow \& Hanson, 1985; Shand, 1982). Indeed, Shand (1982) considered cherological encoding to be the preferred encoding basis for deaf signers of American Sign Language (ASL), regardless of presentation mode. However, recent studies of the encoding strategies used by deaf subjects during reading (Hanson et al,, 1984; Hirsh-Pasek \& Treiman, 1982; Lichtenstein, 1985) have also found evidence for phonological encoding. In these studies, subjects who were considered "good" readers, 
or were more academically successful, were more likely to encode phonologically. Likewise, Hanson (1982) found phonological encoding among deaf subjects when they were required to provide ordered, rather than free, recall. Conrad (1979), Craine (1977), and Hirsh-Pasek and Treiman (1982) found the tendency to encode phonologically to be a function of hearing loss and speech ability. In those studies, subjects with a milder hearing loss and greater speech ability were the most likely to use a phonological code in STM.

Although the above studies have suggested that linguistic stimuli may be encoded in STM in more than one way, they have not defined the critical individual or task variables that determine how a person will encode a particular stimulus. Shand (1982) suggested that early linguistic experience may establish or "fix" a preferred basis for linguistic encoding. However, his study, like most others to date, at least partially confounded the early linguistic experience of subjects (i.e., first-language experience with spoken English or sign) with their sensory capabilities (i.e., hearing or deaf). Conrad (1979), Craine (1977), Hanson et al., (1984), Hirsh-Pasek and Treiman (1982), and Lichtenstein (1985) have suggested that subjects with better hearing, speech, and reading skills are more likely to employ a phonological code than a visual or cherological code when presented with print material. However, in a study with hearing children, Brown (1977b) found evidence that flexible encoding (i.e., either phonological or visual) of printed letters can occur as a function of the probe modality. Brown (1977a) has also found evidence that preschoolers' encoding processes are biased by the characteristics of the input stimuli, in this case visual, even when the stimuli could be recoded phonologically to eliminate the detrimental effects of similar stimulus characteristics on subsequent recall. Taken as a whole, the results of previous studies suggest that rather different types of coding processes may be utilized by different individuals. Generally, deaf subjects appear to use cherological codes for signs and a variety of codes (phonological, cherological, visual, finger-spelling) for print.
Hearing subjects, on the other hand, appear to use phonological codes for the most part, but may utilize visual codes for print in some instances.

Thus, previous research has uncovered instances that suggest that different kinds of encoding can be used by the same person. In the present study, we investigated whether an individual's encoding basis is fixed by the individual's early linguistic experience, as Shand (1982) suggests, or is flexible and biased by stimulus characteristics, as Brown (1977a, 1977b) suggests. We employed groups of subjects, stimuli, and presentation modes with systematically varied characteristics to determine the factors most involved in determining encoding bases. We tested whether encoding was fixed by sensory ability (hearing or deaf) or early linguistic experience (firstlanguage learning), or was flexible and a function of stimulus characteristics.

We utilized stimulus materials and presentation modes that could detect instances of encoding flexibility and better reveal the nature of this flexibility, if it occurred. In addition, efforts were made to determine whether subjects could simultaneously utilize two bases for encoding in STM (phonological and cherological) to enhance recall and eliminate confusions among words in recall lists, whether subjects could select at will an encoding basis that minimized interference effects among recall items, or whether the presentation modality of the stimulus (oral, manual, or bimodal) determined the subjects' basis for encoding.

\section{METHOD}

\section{Subjects}

Six groups of subjects with distinct linguistic backgrounds and auditory status were selected for this study, as shown in Table 1. All subjects were at least 18 years old and were attending or had graduated from a college program. The groups were:

1. Hearing speakers of English (i.e., hearing children of hearing parents) whose first language was spoken English and who were unfamiliar with sign language $\left(\mathrm{HHP}_{1}\right)$. All of these subjects were Georgia State University undergraduate students.

Table 1

Subject Characteristics

\begin{tabular}{|c|c|c|c|c|}
\hline \multirow[b]{2}{*}{ Group } & \multirow[b]{2}{*}{$n$} & \multirow[b]{2}{*}{ Present Auditory Status } & \multicolumn{2}{|c|}{ Linguistic Experience } \\
\hline & & & $\begin{array}{c}\text { First Language } \\
\text { Experience }\end{array}$ & $\begin{array}{c}\text { Second Language } \\
\text { Experience }\end{array}$ \\
\hline $\mathrm{HHP}_{1}$ & 10 & Normal hearing & Spoken English & None \\
\hline $\mathrm{HHP}_{2}$ & 10 & Normal hearing & Spoken English & Sign \\
\hline HDP & 10 & Normal hearing & ASL & $\begin{array}{l}\text { Spoken English } \\
\text { (early in life) }\end{array}$ \\
\hline DAS & 10 & $\begin{array}{l}2 \text { with hearing loss of } 75 \mathrm{~dB} \text { or more } \\
8 \text { with loss of } 90 \mathrm{~dB} \text { or more in better ear }\end{array}$ & Spoken English & Sign \\
\hline $\mathrm{DDP}_{1}$ & 8 & $\begin{array}{l}6 \text { with hearing loss of } 75 \mathrm{~dB} \text { or more } \\
2 \text { with loss of } 90 \mathrm{~dB} \text { or more in better ear }\end{array}$ & ASL & $\begin{array}{l}\text { Spoken English } \\
\text { (fluent speakers) }\end{array}$ \\
\hline $\mathrm{DDP}_{2}$ & 10 & Hearing loss of $90 \mathrm{~dB}$ or more in better ear & ASL & $\begin{array}{l}\text { Spoken English } \\
\text { (nonfluent speakers) }\end{array}$ \\
\hline
\end{tabular}


2. Hearing speakers of English (i.e., hearing children of hearing parents) whose first language was spoken English and who had been using a form of signed English for two years or longer $\left(\mathrm{HHP}_{2}\right)$. All of these subjects were teachers of deaf children.

3. Hearing speakers of English who were first exposed to ASL (i.e., hearing children of deaf signing parents) and whose second language was spoken English (HDP). These subjects were considered fluent in spoken English because they were not identified as needing special classes or speech therapy for spoken English. All of these subjects were sign-language interpreters or professionals working in the field of deaf education.

4. Subjects deafened after age 6 whose first language was spoken English (i.e., postlingually deaf children of hearing parents) who had been using either ASL or signed English for two years or longer (DAS). One of these subjects was a medical technologist, 2 were teachers of deaf children, and 7 were students at the National Technical Institute for the Deaf (NTID). Two had hearing losses of $75 \mathrm{~dB}$ or more in the better ear and 8 had losses of $90 \mathrm{~dB}$ or more in the better ear. Thus, all were severely to profoundly hearing-impaired.

5. Congenitally deaf subjects whose first language was ASL (i.e., deaf children of deaf parents) and who had acquired intelligible spoken English $\left(\mathrm{DDP}_{1}\right)$. This was determined by a score of 3.65 on the speech intelligibility test used at NTID (Johnson, 1976). The intelligibility of the subjects' speech indicated that most of the productive phonological system of English had been acquired. All of these subjects were NTID students. Six had hearing losses of $75 \mathrm{~dB}$ or more in the better ear and 2 had losses of $90 \mathrm{~dB}$ or more in the better ear. Thus, all of these subjects were severely to profoundly hearing-impaired.

6. Congenitally deaf subjects whose first language was ASL (i.e., deaf children of deaf parents) who had not acquired spoken English fluency $\left(\mathrm{DDP}_{2}\right)$. This was determined by a score of $1.0-1.5$ on the speech intelligibility test used at NTID. Such a score indicated the subject's speech was unintelligible. Thus, the productive phonological system of spoken English had not been acquired. All of these subjects were NTID students and had hearing losses of $90 \mathrm{~dB}$ or more in the better ear. Thus, all were profoundly hearingimpaired.

Measures of lipreading ability were not taken for any subjects in this study.

As shown in Table 1, only $8 \mathrm{DDP}_{1}$ subjects were obtainable because of their scarcity in the population. Out of approximately 1,000 students at NTID, only 11 possessed the DDP, characteristics. This is approximately $1 \%$ of that institution's population and, thus, such subjects constitute an even smaller proportion of the general human population, hearing and deaf.

In order to obtain adequate numbers of scarce types of subjects, all deaf subjects at NTID were paid $\$ 10$ for their participation. All other subjects were volunteers who received no reimbursement.

\section{Materials}

A set of items was assembled for a short practice session. These items were selected from signs that have the same commonly used English translation in signed English and ASL. The items used for the practice session were VERY, BOY, TREE, STOP, and DOLLAR.

For the experimental session, cherologically related, phonologically related, and control lists were assembled from the items utilized by Shand (1982), to provide a common basis for comparing the results of the two studies. The signs associated with these items (listed in the appendix) are the same in signed English and ASL. Problems found with printed presentations of Shand's items (Hanson \& Lichtenstein, 1986) were avoided in this study by not using such a presentation mode

In Shand's (1982) study, the relatedness of items was determined by their shared linguistic parameters and also by experimental and intuitive methods of research. Precise definitions of phonologically similar, cherologically similar, and unrelated items have not appeared in the literature to date. Consequently, we developed a set of parameters for item similarity in conjunction with the present investigation, drawing upon previously employed conventions whenever possible.

Criteria for phonological similarity. The general notion of sound-based similarity used in earlier studies was adapted to delineate phonological similarity in this study. Generally, errors and targets that were said to be phonologically related either rhymed or alliterated (Bellugi et al., 1975; Fromkin, 1980). Many previous studies' related pairs also contained the same number of syllables and stress pattern, (i.e., metric value). The preceding three criteria have not only been prevalent in psycholinguistic research, but have also been important constituents of poetry for centuries. As such, these parameters appear to be critical determinants of human perception, encoding, and production of sound-based similarity. We included a fourth parameter, canonical form (i.e., the order of consonants and vowels in a word), to allow a comparison of the general shape of spoken words. For example, using only the first three parameters described, the words brew and boo are as similar as are boo and boo. Each rhymes, alliterates, and has the same metric value. If canonical form is included, then brew (consonantconsonant-vowel) and boo (consonant-vowel), which are not canonically identical, can be considered less phonologically similar than can boo and boo, which are identical.

Two additional parameters of phonological similarity used in this study were near-rhyme and near-alliteration. Near-rhyme refers to the use of sounds which differ in only one production parameter in the final position of words. Near-alliteration refers to the recurrence of sounds which differ in only one production parameter in the initial position of words.

Each possible combination of items in this study was given a phonological similarity score. All shared parameters were worth two points except for near-rhyme and near-alliteration, which were given one point. For example, the spoken words show and sew rhyme, have the same metric shape, and the same canonical form. They are also near-alliterations. This pair received a score of 7 . The spoken words jealous and new share none of these features and therefore received a score of 0 . Each list that was considered phonologically related contained two or more pairs of items whose phonological similarity scores were 4 or higher.

Criteria for cherological similarity. Shand (1982) used the parameters of movement, location, and handshape (first described by Stokoe, 1960), as well as sign similarity judgments by deaf native signers, to rate cherological similarity. Newkirk, Klima, Pederson, and Bellugi (1980) considered the parameters of movement, location, and handshape as major parameters because they distinguish very large classes of signs. In contrast, contacting region of the hand, orientation of the palm, and hand arrangement (the number of hands active or passive in a sign) were considered minor parameters by Newkirk et al. because they distinguish very small sets of minimal pairs (i.e., lexical items that differ in only one structural feature). It is common in ASL for minimal pairs to be distinguished by one of the major parameters (e.g., the signs JAPANESE and CHINESE differ only in handshape) and it is rare for minimal pairs to differ in only a minor parameter. One example of the latter situation is YELLOW and PLAY, which differ only in the number of hands involved in the signs. In the present study, we based our rating of cherological similarity on the three major parameters used by Shand and on the additional minor parameters described by Newkirk et al.

For scoring purposes, sharing of a similar major parameter was given a score of 2 and sharing of a minor parameter was given a score of 1 . For example, CANDY and APPLE share two major parameters and all minor parameters; there is only a handshape difference between the two signs. The cherological similarity score for this pair would therefore be 7 . In contrast, ONION and 
THROUGH share no cherological parameters; the score for this pair would be 0 . Each list that was considered cherologically similar contained two or more pairs of items whose cherological similarity scores were 4 or higher. No pairs of items in this study received a score of 4 or above for both phonological and cherological similarity.

Criteria for unrelated items. Items were considered unrelated if they received phonological and cherological scores of 3 or less. Each control list consisted of items that, when paired, had phonological and cherological scores of 3 or less. In all, 120 pairs could be generated from the 16 words used in this study. Twenty-two of these pairs had phonologically similar items, 28 had cherologically similar items, and 70 had unrelated items. The proportions of each type of pair were $.183, .233$, and .583 , respectively. These proportions were used to calculate the expected frequencies for a subsequent chi-square analysis of intrusion errors.

\section{Procedure}

In both the practice and experimental sessions, the stimuli were presented via videotape in three different modalities-oral, manual, and simultaneous - by a hearing adult born to deaf parents (a native user of ASL) who was also a fluent speaker of English. The presenter was not informed about the hypotheses being investigated. Stimulus lists consisted of five items each. This was identical to Shand's (1982) list length. The subjects were tested either individually or in groups of 2 . When in groups of 2 , the subjects were not allowed to communicate with each other during any part of the session.

During the task instructions, the experimenter communicated using simultaneous communication. The subjects were first asked to read the directions for the practice session, and then viewed these directions signed and spoken by the experimenter on a previously made videotape. The five stimulus items for the practice session were then introduced to the subjects in speech, signs, and print simultaneously. That is, as the presenter signed and spoke each stimulus item during the introductory section of the experiment, the printed word also appeared on the screen. The subjects were then instructed to watch the videotape for a person who would sign, speak, or speak and sign simultaneously.

We used a paradigm similar to that of Baddeley (1966) and Shand (1982). The subjects were told that five items would be presented and they should write the English words for those items in order on a response sheet after the presenter provided the five stimuli. The television screen was then blacked out for $30 \mathrm{sec}$ while the subjects responded. After about $23 \mathrm{sec}$, color bars appeared on the screen to alert the subjects to be ready for the next presentation. This procedure was used for all three lists in the practice set. Written responses were required in order to provide a "neutral" or common response for all subjects that would not confound the results with different response modes for different subjects. With just five simple words and no grammar required for recall, the writtenresponse mode was judged to be easily usable by all six groups of college-educated subjects. Furthermore, even if the groups differed in their ability to use the written-response mode, it would not seriously impede this investigation. The principal questions of interest to the study did not concern absolute performance differences between groups but, rather, differences within groups under varying stimulus conditions.

After the practice set, the subjects were asked to read the directions for the experimental session and then viewed these directions signed and spoken on videotape. The stimulus items for the experimental session were introduced in speech, signs, and print simultaneously on the videotape. The experimental session then began. In previous studies (e.g., Baddeley, 1966; Shand, 1982), the subjects were allowed to see the printed English words for all the stimulus items displayed in the testing room during the experiment. Such a procedure was not included in this experiment to minimize the likelihood that the subjects would utilize other mnemonic strategies, such as referring to the displayed words as they were presented and subsequently using the location of these words to aid recall.

The lists were presented in six sets of nine in speech, signs, or speech and signs simultaneously. After each set, the subjects had a 5-min rest period. After approximately $4.5 \mathrm{~min}$, color bars appeared on the screen to alert the subjects that the next set would begin shortly.

Six phonologically related, cherologically related, and control lists were presented in each mode of presentation. Therefore, with five items per list, there were a total of 30 items ( 6 lists $\times 5$ items) to be recalled for each list type in each presentation mode. In all, there were 18 lists per presentation mode. This yielded 54 lists (18 lists $\times 3$ presentation modes) that were presented to each subject. The list types and presentation modes were balanced across all presentations so that certain list types and presentation modes did not all appear either very early or very late in the experimental session.

\section{RESULTS AND DISCUSSION}

\section{Mode of Analysis}

A $6 \times 3 \times 3$ repeated measures design was employed in this study. There was one between-subjects factor (auditory-linguistic background/status) and two withinsubjects factors (presentation mode and list type). Both position-scoring and intrusion-error analysis were done. In position scoring, a subject's response was considered correct if it was the correct form and in the same position as in the stimulus list (Shand, 1982). Intrusion errors were defined on the basis of the criteria used by Bellugi et al. (1975):

A response that was incorrect for a position on the specific list in question but was:

a) not a misplaced item for that list;

b) not a repetition of a response made to another item on the list;

c) not an occurrence of an item from the immediately prior list. (p. 106)

Means and standard deviations computed from position scoring for recall performance appear in Table 2. Analyses of variance (ANOVAs) incorporating a set of planned comparisons and tests for simple main effects were used to examine the recall performance in relation to subject characteristics, list types, and presentation modes. Intrusion errors were noted and analyzed to determine if there was significantly more of any type of error for each subject group within each presentation mode. Main effects and interactions from the analyses of variance will be reported first.

\section{Recall Accuracy}

Auditory-linguistic group differences. The ANOVA revealed a significant main effect for group membership $[F(5,52)=13.45, p<.001]$, with groups ranked as follows from highest to lowest: $\mathrm{HHP}_{2}(M=240.6)$, HDP $(M=228.2), \mathrm{DDP}_{1}(M=197.49)$, DAS $(M=187.8)$, $\mathrm{HHP}_{1}(M=173.4)$, and $\mathrm{DDP}_{2}(M=160)$. The sources of this main effect of groups merit additional investigation by other studies. However, as pointed out earlier, 
Table 2

Mean Number Correct for Recall Performance*

\begin{tabular}{|c|c|c|c|c|c|c|c|c|c|c|}
\hline \multirow[b]{3}{*}{ List Type } & \multicolumn{9}{|c|}{ Mode } & \multirow[b]{3}{*}{ Margir } \\
\hline & \multicolumn{3}{|c|}{ Oral } & \multicolumn{3}{|c|}{ Manual } & \multicolumn{3}{|c|}{ Simultaneous } & \\
\hline & Phon & Cher & Control & Phon & Cher & Control & Phon & Cher & Control & \\
\hline $\mathrm{HHP}_{1}$ & & & & & & & & & & \\
\hline$M$ & 22.4 & 23.6 & 23.4 & 16.5 & 7.5 & 13.3 & 20.7 & 23.0 & 23.0 & 173.4 \\
\hline$S D$ & 3.8 & 2.3 & 3.4 & 3.2 & 2.9 & 4.2 & 3.6 & 4.4 & 5.1 & \\
\hline Total & \multicolumn{3}{|c|}{69.3} & \multicolumn{3}{|c|}{37.3} & \multicolumn{3}{|c|}{66.7} & \\
\hline \multicolumn{11}{|l|}{$\mathbf{H H P}_{2}$} \\
\hline$M$ & 27.7 & 27.5 & 26.5 & 25.7 & 24.9 & 25.8 & 27.2 & 28.3 & 27.0 & 240.6 \\
\hline$S D$ & 1.3 & 2.1 & 2.9 & 5.4 & 4.3 & 4.1 & 2.1 & 2.3 & 2.7 & \\
\hline Total & \multicolumn{3}{|c|}{81.7} & \multicolumn{3}{|c|}{76.4} & \multicolumn{3}{|c|}{82.5} & \\
\hline \multicolumn{11}{|l|}{ HDP } \\
\hline$M$ & 24.7 & 26.4 & 27.4 & 23.7 & 22.5 & 24.9 & 25.8 & 26.2 & 26.6 & 228.2 \\
\hline$S D$ & 5.1 & 3.3 & 2.1 & 5.1 & 5.2 & 4.9 & 3.9 & 3.1 & 2.7 & \\
\hline Total & \multicolumn{3}{|c|}{78.5} & \multicolumn{3}{|c|}{71.1} & \multicolumn{3}{|c|}{78.6} & \\
\hline \multicolumn{11}{|l|}{ DAS } \\
\hline $\boldsymbol{M}$ & 16.5 & 22.9 & 20.0 & 19.8 & 18.0 & 20.0 & 23.9 & 22.5 & 24.2 & 187.8 \\
\hline$S D$ & 8.2 & 5.8 & 7.1 & 6.1 & 6.6 & 6.5 & 4.7 & 5.6 & 2.8 & \\
\hline Total & \multicolumn{3}{|c|}{59.4} & \multicolumn{3}{|c|}{57.8} & \multicolumn{3}{|c|}{70.6} & \\
\hline \multicolumn{11}{|l|}{ DDP, } \\
\hline$M$ & 19.25 & 21.25 & 20.0 & 21.25 & 19.75 & 23.75 & 24.75 & 25.37 & 22.12 & 197.49 \\
\hline$S D$ & 5.3 & 6.4 & 6.0 & 4.3 & 5.2 & 3.9 & 3.5 & 2.5 & 5.6 & \\
\hline Total & \multicolumn{3}{|c|}{60.5} & \multicolumn{3}{|c|}{64.7} & \multicolumn{3}{|c|}{72.2} & \\
\hline \multicolumn{11}{|l|}{$\mathrm{DDP}_{2}$} \\
\hline$M$ & 6.6 & 12.0 & 11.1 & 22.8 & 15.5 & 21.8 & 22.8 & 29.6 & 17.8 & 160.0 \\
\hline$S D$ & 3.8 & 5.1 & 4.4 & 3.6 & 5.4 & 5.3 & 5.8 & 5.2 & 6.3 & \\
\hline Total & \multicolumn{3}{|c|}{29.7} & \multicolumn{3}{|c|}{60.1} & \multicolumn{3}{|c|}{70.2} & \\
\hline Margin & & & & & & & & & & \\
\hline$M$ & 19.53 & 22.31 & 21.44 & 21.63 & 17.96 & 21.51 & 24.17 & 24.12 & 23.5 & 196.17 \\
\hline Total & & 63.1 & & & 61.2 & & & 73.5 & & \\
\hline
\end{tabular}

they do not, in themselves, bear directly on the central issues of this study (i.e., the within group differences indicative of STM encoding processes); consequently, they will be only briefly discussed here.

Three basic factors appear at least partially responsible for the quantitative recall differences between groups: perceptual access to the stimuli, experience with the stimuli, and fluency with the stimuli. By considering each of these factors, the rank order of the subject groups' recall performance can be largely explained. Groups $\mathrm{HHP}_{2}$ and HDP (ranked 1 and 2, respectively) had full perceptual access to each type of stimulus, a great deal of experience with each type of stimulus, and high levels of fluency with each stimulus type. In comparison, Groups DDP $_{1}$ and DAS (ranked 3 and 4, respectively) had less access and fluency with the oral stimuli than did Groups $\mathrm{HHP}_{2}$ and HDP, which explains their lower scores. Group DAS also had less experience with the manual and simultaneous stimuli than did Group DDP ${ }_{1}$, which accounts for the former group's ranking of fourth compared to Group $\mathrm{DDP}_{1}$ 's ranking of third. An examination of the mean recall for each modality in Table 2 supports this contention. For Group $\mathrm{HHP}_{1}$, the limited experience and fluency with the manual stimuli severely hindered recall of this information and lowered the overall recall scores greatly, which accounts for this group's ranking of fifth. For Group $\mathrm{HHP}_{1}$, the experiment was more of a learning experience than a test in the manual and simultaneous presentation modes. Indeed, it is surprising that any recall occurred at all in the manual-presentation situations. It is possible that the simultaneous-presentation situations allowed Group HHP, the opportunity to learn English equivalents for the manually presented signs, as the spoken word was paired with it simultaneously during presentation. The recall performance of Group $\mathrm{DDP}_{2}$ was the lowest of all six groups and can be attributed to each of the factors mentioned above. Particularly, Group DDP $_{2}$ had limited perceptual access to the oral stimuli, very little "successful" experience with it (however, they had had experience with it via their educational experiences), and very little oral fluency. As mentioned above, these results merit further investigation but do not directly address the main issues of this study. Rather, the interactions of groups with list types and presentation modes will be further explored to investigate their influences on linguistic encoding in STM.

List-type differences. The ANOVA revealed no significant main effect for list type. The mean number of 
correct responses was very similar across list types: 65.33 , 64.39 , and 66.45 for phonological, cherological, and control lists, respectively. Had one list type been recalled significantly less well than the others, one universal coding process would have been suggested. The lack of differences between list types obtained here conforms with other research (e.g., Lichtenstein, 1985) that suggests the existence of more than one basis for linguistic encoding in STM. This topic will be directly addressed in the discussion of the mode $\times$ list interaction.

Enhanced encoding evidence. The main effect of mode of presentation was significant $[F(2,52)=30.83, p<$ $.001]$. Simultaneous presentations $(M=73.47, S D=$ 3.98) were recalled better than were oral presentations $(M=63.18, S D=4.31)$, which were recalled somewhat better than were manual presentations $(M=61.24, S D$ $=4.78$ ). Apparently, bimodal presentations facilitated substantially better stimulus encoding than did unimodal presentations, regardless of the specific type of single modality used. Such a statement must be qualified, however, due to the mode $\times$ group interaction.

The interaction between mode and group was significant $[F(1,104)=18.56, p<.001]$, a result of importance for addressing the possibility of enhanced encoding of bimodal stimulus presentation. To test this hypothesis, groups of subjects were contrasted according to whether they had only speech experience (HHP $\left.{ }_{1}\right)$ or had both sign and speech experience (i.e., all groups except $\mathrm{HHP}_{1}$ ), signified as ALL-HHP ${ }_{1}$. The possibility that subjects with sign and speech experience recalled simultaneous presentation significantly better than oral or manual presentation, and that subjects with only speech experience did not benefit from simultaneous presentation in a similar manner, can then be addressed.

As can be seen from Table 2, all groups except HHP, scored highest in the simultaneous-presentation condition. For ALL-HHP ${ }_{1}$, recall under simultaneous presentations was significantly higher than recall under oral $[F(1,104)$ $=144.89, p<.001]$ and manual $[F(1,104)=54.25$, $p<.001]$ presentations. These results support the hypothesis that enhanced encoding for bimodal presentations exists and that subjects with sign and speech experience are capable of using it to increase recall.

A closer look at the individual groups comprising ALL$\mathrm{HHP}_{1}$ indicates that, although the simultaneous mode was the best presentation mode for all of them, the magnitude of this effect varied considerably. The largest difference between means within ALL-HHP ${ }_{1}$ appeared for deaf subjects. These subjects recalled much more information in the simultaneous condition than in either the oral or the manual condition. For the hearing subjects comprising Groups $\mathrm{HHP}_{2}$ and $\mathrm{HDP}$, simultaneous-condition means were much higher than manual-condition means, but were only slightly higher than oral-condition means. This result may be due to a ceiling effect; both groups obtained approximately $90 \%$ correct in each modality. A more strenuous test of memory capacity may have allowed a more apparent enhanced encoding effect of the simultaneouspresentation condition for Groups $\mathrm{HHP}_{2}$ and HDP.
With regard to presentations in just one modality, ALL$\mathrm{HHP}_{1}$ subjects recalled significantly more information under manual conditions than under oral conditions $[F(1,104)=21.82, p<.001]$. However, this result does not accurately reflect the oral versus manual performance of all the constituent subgroups of subjects. Table 2 indicates that the recall of Groups $\mathrm{DDP}_{1}$ and $\mathrm{DDP}_{2}$ was generally higher for manual presentations, and the recall of Groups $\mathrm{HHP}_{2}$, HDP, and DAS tended to be higher for oral presentations.

The results for Groups DDP $1, \mathrm{DDP}_{2}, \mathrm{HHP}_{2}$, and HDP are not totally surprising, due to the relative inaccessibility of the oral stimuli for the deaf subjects and the extensive experience the hearing subjects had had with oral communication. Even with deaf parents, Group HDP subjects had grown up learning to process in predominately oral educational, social, and mass media systems. The finding that Group DAS scored higher on oral presentations than on manual presentations is surprising, however, considering the inaccessibility of the oral stimuli, compared with the manual stimuli, for these subjects. This finding supports the view that early linguistic experience has an effect on the quantitative aspects of recall performance that is not totally lost even with radical subsequent changes in that experience. Future research with DAS subjects on this phenomenon is needed to elucidate the nature of its operation further. It is possible that the early oral experience of these subjects reinforced special attention to lip movements, on which these individuals can capitalize for verbal encoding.

The subjects that lacked training in manual communication (Group $\mathrm{HHP}_{1}$ ) recalled orally presented stimuli significantly better than either simultaneously presented $[F(1,104)=5.14, p<.05]$ or manually presented $[F(1,104)=265.06, p<.001]$ stimuli. Simultaneously presented stimuli were, in turn, recalled significantly better than those that were manually presented $[F(1,104)=$ $196.35, p<.001]$. These results fit well with the hypothesis that subjects without both speech and sign experience cannot make use of the dual information available in bimodal simultaneous presentation to enhance recall significantly. In fact, simultaneous stimuli were recalled less well than were unimodal oral stimuli. This may be due to the sign-language information in the simultaneous signal acting as a distractor for these "naive" signers. Their lack of facility with sign language was further emphasized by their poor performance on manual presentations. It is interesting to note, however, that Group HHP, subjects showed some degree of recall for manually presented items, which suggests that learning occurred for these subjects during the experimental session. The simultaneous presentations could have allowed the subjects to learn the oral label/word for the manual sign. That their recall performance for simultaneous presentation was higher than their recall for manual presentation is presumably due to the oral part of the simultaneous signal. The preceding analyses suggest that the ability to utilize enhanced encoding for dual stimuli is a function of total linguistic experience. The ALL-HHP, 
subjects were capable of enhanced encoding for bimodal stimuli, and the subjects with only speech experience (Group HHP $_{1}$ ) were not able to utilize the dual stimuli in the same manner. Furthermore, subject groups did not interact significantly with list type. If encoding bases were a function of hearing ability or early linguistic experience alone, a significant interaction of list and groups should have occurred.

Flexible encoding evidence. The interaction between presentation mode and list was significant $[F(4,208)=$ $20.47, p<.001]$. This finding warrants further analysis of the data for evidence of encoding flexibility, that is, the change of codes in response to various stimulus conditions. To investigate this phenomenon, a detailed examination of recall trends was conducted in conjunction with the tests for simple main effects of list type within presentation mode.

If coding were flexible, yet biased by stimulus characteristics, subjects would be expected to exhibit lower recall performance on phonologically related (and thus phonologically confusable) lists as compared to cherological and control lists in the oral-presentation condition. In accordance with the flexible-encoding hypothesis, statistical tests of simple main effects showed that phonological lists were recalled significantly less well than were cherological lists $[F(4,208)=6.85, p<.01]$ and control lists $[F(4,208)=3.09, p<.05]$. In fact, this trend occurred for all groups except Group $\mathrm{HHP}_{2}$ (see Table 2); that group scored lowest on control lists in the oralpresentation condition. However, the differences between list types were nonsignificant for Group $\mathrm{HHP}_{2}$, which scored right around $90 \%$ correct on all list types in the oral condition. Thus, Group HHP ${ }_{2}$ 's departure from the list-type pattern demonstrated by the other groups may be attributed to ceiling effects. A more strenuous test of the memory capacity of Group $\mathrm{HHP}_{2}$ may have uncovered the expected decrement in recall of phonologically related lists.

In the manual-presentation condition, the expected trend to support the flexible-encoding hypothesis stated above would be for recall of cherological lists to be lower than recall of phonological and control lists. This trend was consistently found for all subject groups (see Table 2). Tests of simple main effects showed that cherological lists were recalled significantly less well than were phonological lists $[F(4,208)=11.76, p<.01]$ and control lists $[F(4,208)=10.12, p<.01]$. This effect was strongest for Group $\mathrm{HHP}_{1}$, which was comprised of nonfluent signers. A strong effect was also seen for Group $\mathrm{DDP}_{2}$, which was comprised of fluent signers (see Table 2). Further investigation is needed to understand the factors in fluencing this result for Group $\mathrm{DDP}_{2}$ fully.

If flexible encoding can occur, the simultaneous condition should produce a flat distribution of scores across list types. In addition, the presence of speech and sign simultaneously should alleviate the negative effects that list type has on recall within a unimodal presentation for all subject groups. This is generally what occurred.
Groups HDP and HHP, scored lowest on phonological lists, Group DAS scored lowest on cherological lists, and Groups $\mathrm{HHP}_{2}, \mathrm{DDP}_{1}$, and $\mathrm{DDP}_{2}$ scored lowest on control lists (see Table 2). Tests of simple main effects revealed no significant difference between any list types within the simultaneous mode. It is suggested that the addition of sign to the accompanying speech alleviated the negative effects of phonological similarity for some subjects and, conversely, the speech accompanying the sign alleviated the negative effects of cherological similarity for other subjects.

The three-way interaction between mode, list, and group was significant $[F(20,208)=3.68, p<.001]$, which indicates that list types were differentially recalled by different groups in different modalities. This effect is most likely due to fluency with the stimuli presented. For example, the mean recall for orally presented phonological lists was 22.4 for Group HHP (fluent speakers/ $_{1}$ nonfluent signers) and 6.6 for Group $\mathrm{DDP}_{2}$ (nonfluent speakers/fluent signers). By examining Table 2 , it becomes evident that differences exist between groups which appear, once again, to be a function of accessibility to the stimuli, experience with the stimuli, and fluency with the stimuli presented in that modality. Such results indicate differences in recall between groups rather than within groups. As discussed earlier, within-group differences directly address the issues under study in this experiment. The between-group differences, although not of specific interest for this particular study, do merit further investigation in future research.

\section{Intrusion Errors}

In addition to recall-accuracy scores, we examined patterns of intrusion errors bearing on the major hypotheses of this study. The results of the chi-square analyses, applied to these data, are presented in Table 3. An intrusion was considered to be phonologically similar to the target if the phonological similarity score for the pair was 4 or higher. Likewise, cherologically similar intrusiontarget pairs had a cherological similarity score of 4 or higher. The chi-square analysis revealed significant differences in the types of intrusion errors that occurred as a function of presentation mode in nearly every analysis done. In the oral-presentation conditions, all groups of subjects made significantly more phonological intrusions than cherological or unrelated intrusions. In the manualpresentation condition, all groups of subjects made significantly more cherological intrusions than phonological or unrelated intrusions. In the simultaneous condition, all groups except Groups $\mathrm{DDP}_{1}$ and $\mathrm{DDP}_{2}$ made significantly more phonological intrusions than cherological or unrelated intrusions.

The results for oral and manual presentations further support the hypothesis that coding is flexible and is biased by the formal characteristics of the incoming stimuli. This appears to be true for all subjects. The intrusion errors in the simultaneous-presentation condition 
Table 3

\begin{tabular}{|c|c|c|c|c|c|c|c|c|c|c|c|c|}
\hline \multicolumn{13}{|c|}{$\begin{array}{c}\text { Chi-Square Analysis of Intrusion Errors by } \\
\text { Presentation Mode }\end{array}$} \\
\hline & \multicolumn{4}{|c|}{ Oral Presentation } & \multicolumn{4}{|c|}{ Manual Presentation } & \multicolumn{4}{|c|}{ Simultaneous Presentation } \\
\hline & Phon & Cher & Unr & $x^{2}$ & Phon & Cher & Unr & $x^{2}$ & Phon & Cher & Unr & $x^{2}$ \\
\hline $\mathrm{HHP}_{1}$ & 21 & 2 & 4 & $68.9^{*}$ & 19 & 63 & 20 & $90.96^{*}$ & 33 & 1 & 8 & $102.36^{*}$ \\
\hline $\mathrm{HHP}_{2}$ & 7 & 0 & 1 & $25.67^{*}$ & 4 & 14 & 3 & $23.93 *$ & 8 & 0 & 2 & $25.64^{*}$ \\
\hline HDP & 11 & 1 & 1 & $38.1^{*}$ & 4 & 24 & $\mathbf{0}$ & $63.44^{*}$ & 11 & 1 & 4 & $27.29 *$ \\
\hline DAS & 45 & 8 & 10 & $119.66^{*}$ & 6 & 29 & 7 & $50.58^{*}$ & 12 & 4 & 9 & $14.75^{*}$ \\
\hline $\mathrm{DDP}_{1}$ & 39 & 10 & 6 & $103.47^{*}$ & 4 & 15 & 3 & $26.55^{*}$ & 4 & 3 & 5 & 2.97 \\
\hline $\mathrm{DDP}_{2}$ & 38 & 9 & 13 & $100.51 *$ & 5 & 27 & 4 & $55.43^{*}$ & 5 & 6 & 10 & 1.00 \\
\hline
\end{tabular}

Note-Phon $=$ phonological, Cher $=$ cherological, Unr $=$ unrelated. ${ }^{*} p \leq .001$.

lend support to the possibility that, when stimulus characteristics are mixed, early linguistic experience may bias how a person encodes. All groups that had significantly more phonological intrusions had early experience with speech. Even Group HDP, most likely, had a great amount of exposure to spoken English from television, radio, and hearing friends or neighbors. It is possible that the simultaneous communication experience of Groups $\mathrm{DDP}_{1}$ and $\mathrm{DDP}_{2}$, primarily through their contact with hearing signers who dominate school settings for the deaf, allowed them to reach a high level of skill for processing this mode of communication. Thus, they may not favor one modality over the other greatly when simultaneously presented. Rather, one signal complements the other to alleviate any negative confusion effects inherent in the stimuli.

\section{SUMMARY AND CONCLUSIONS}

In this study, we sought to delineate the encoding bases utilized in STM more clearly, and thus provide a more complete picture of STM processes. Past research had used homogeneous subject groupings. By studying subjects who varied in sensory and experiential factors, this study allowed a broader view to be obtained.

With regard to presentation formats, the utilization of "primary" modes (i.e., spoken and signed languages, the codes of which are not based on another established language code) allowed us to delineate the process of linguistic encoding in STM more accurately than has been done in many past studies that utilized a "secondary" mode of communication (such as print for which the language code is derived from a spoken language). By presenting stimuli in a primary mode and then in their accompanying secondary mode (e.g., the speech- and print-presentation formats utilized by Baddeley, 1966, or the sign and print formats of Shand, 1982), past research has actually been studying the same encoding process, due to the dependence of the secondary code on the primary code.

Legge and Kaminska's (1975) response-monitoring modality hypothesis further supports the contention that some previous studies may have arrived at questionable conclusions because primary and secondary codes were confounded. Legge and Kaminska have suggested that items are stored in STM in the language or code of the modal- ity through which the customary responses to these items are normally monitored. Thus, as print is presented, it is stored in the code of the modality through which it is normally monitored, which is phonological for most hearing and mildly hearing-impaired subjects, and cherological for most deaf subjects. As a result of investigating this dependence of secondary-mode information on primarymode information for STM encoding, claims that encoding bases are unaffected by presentation mode have sometimes been made without rigorous comparisons of true differences in primary modes. By utilizing independent primary modes in this study, we found that presentation format is indeed of importance in determining how information is encoded.

The results of the present study suggest that the qualitative parameters of STM encoding (how a person encodes) are not strictly fixed by subject characteristics, but are flexible and are influenced by the formal characteristics of the incoming stimuli. Regardless of a subject's hearing ability or early linguistic experience, recall performance was affected in the same way by the same list characteristics within the same unimodal primarypresentation modes. For oral presentations, for example, phonologically related lists were recalled significantly less well than were other list types. Significantly more phonological intrusions also occurred in oral-presentation conditions. For manual presentations, cherologically related lists were recalled significantly less well than were other types of lists. Significantly more cherological intrusions also occurred in manual-presentation conditions. These results provide strong evidence that coding in STM is biased by the formal characteristics of the stimuli. Items that are formally similar are more easily confused and, thus, are recalled less well and intrude in each others' recall. Furthermore, Camden et al. (1982) also found evidence that STM recognition can be biased by the input stimuli when a secondary mode of information, in their case print, is presented.

For simultaneous presentations, there were no differences between recall of list types. This may be due to the presence of both phonological and cherological information such that one alleviates, or allows subjects to compensate for, the negative effects of the other in the recall of phonologically or cherologically related lists. Levy and Craik (1975) found evidence that such compensation can occur when both phonological and semantic information 
are available. However, the significantly larger number of phonological intrusions made by subjects with early speech experience suggests that when stimulus characteristics are bimodal and simultaneously mixed, early linguistic experience may, to some extent, bias the encoding bases used by the subject.

It may be argued that the methods used in this study tapped the perceptual abilities of some subject groups, rather than strictly examined STM encoding and the stimulus-biasing effect described above. For example, hearing subjects with no sign experience may have been consistently perceiving cherological stimuli erroneously and storing them in a phonological code in STM, rather than cherologically as the study suggests. Admittedly, no perceptual tests were given and, although this possibility may account for the lower recall performance of nonsigning subjects, it does not account for the intrusion errors that occurred. These intrusion errors were primarily cherologically related to the targets. Had the sign APPLE been misperceived as CANDY and stored phonologically as "candy," the subsequent accurate recall of the misperceived CANDY would appear as an intrusion error. However, due to the memory load involved for these hearing nonsigners, one would expect a great number of intrusion errors. These did occur, as Group HHP made $_{1}$ 102 intrusions in the manual mode, compared to an average of 29.8 intrusions for all other groups. Thus, a misperceived and phonologically stored CANDY would have been erroneously recalled as "handy" or "dandy." However, such phonological intrusion errors rarely occurred. Rather, cherological intrusions such as ONION (for the target APPLE) occurred with great frequency. Thus, even if a sign was misperceived, cherological information was being held in STM rather than a phonological translation of a misperceived sign.

The results of this study also suggest that the quantitative parameters of linguistic encoding (how much a person encodes and recalls) are primarily a function of total linguistic experience and the presentation mode. For subjects with sign and speech experience, enhanced encoding can occur when dual stimuli are presented via bimodal simultaneous presentation in the form of speech and sign. Recall of bimodal simultaneous presentation was significantly higher than recall of unimodal presentation of oral or manual stimuli for these subjects. This result is of importance for educators of deaf students to consider as they discuss and implement the best way to communicate with deaf students in classroom/learning environments.

For subjects with only speech experience, the presentation mode and familiarity with the stimuli presented in the particular mode (i.e., linguistic experience with these forms) also determined how much was recalled. These subjects recalled significantly more in the oral-presentation condition than in other presentation conditions. Recall in the simultaneous-presentation condition was also significantly higher than recall in the manual-presentation condition. Thus, the subjects' familiarity with the stimuli directly parallelled their recall performance for the stimuli. The more familiar they were with the stimulus characteristics, the more they recalled.

In summary, it may be stated that STM encoding for unimodal information is flexible to the extent that different codes may be used by all subjects, but it is fixed in the sense that the form of the incoming stimuli, rather than subject characteristics, bias which code is utilized. For bimodal presentations, it appears that early linguistic experience may bias the subject to depend on the code that dominated that early experience. The quantitative aspects of STM encoding appear to be affected by the subjects' characteristics, being primarily a function of their perceptual access to the stimuli, total linguistic experience with the stimuli, and fluency with the stimuli.

\section{REFERENCES}

Agrawal, R., \& Sarkaria, T. K. (1981). Some variables in the efficacy of pictorial encoding in STM. Personality Study \& Group Behavior, 1, 39-49.

Atkinson, R., \& Shiffrin, R. (1968). Human memory: A proposed system and its control processes. In K. Spence \& J. Spence (Eds.) The psychology of learning and motivation (Vol. 2, pp. 89-195). New York: Academic Press

BADDELEY, A. D. (1966). Short-term memory for word sequences as a function of acoustic, semantic, and formal similarity. Quarterly Journal of Experimental Psychology, 18, 362-365.

BadDeley, A. D. (1972). Retrieval rules and semantic encoding in shortterm memory. Psychological Bulletin, 78, 379-385.

BadDeley, A. D. (1976). The psychology of memory. New York: Basic Books.

Bellugl, U., Klima, E., \& Siple, P. (1975). Remembering in signs. Cognition: International Journal of Cognitive Psychology, 3, 93-125.

Bellugi, U., \& SiPLE, P. (1974). Remembering with and without words. In F. Bressen (Ed.), Current problems in psycholinguistics (pp. 215 236). Paris: CNRS

Brown, M. R. (1977a). An examination of visual and verbal coding processes in preschool children. Child Development, 48, 38-45.

Brown, M. R. (1977b). Visual and phonetic memory in preschool children: Evidence for coding flexibility. Perceptual \& Motor Skills, 45, 1043-1050.

Camden, C., Motley, M., BaArs, B. J. (1982). Cognitive encoding processes: Evidence for a graphemically based short-term memory. Human Communication Research, 8, 327-337.

Conrad, R. (1962). Acoustic confusions and memory span for words. Nature, 197, 1029-1030.

Conrad, R. (1964). Acoustic confusions in immediate memory. British Journal of Psychology, 55, 75-84.

ConRAD, R. (1971). The chronology of the development of covert speech in children. Developmental Psychology, 5, 398-405.

ConRaD, R. (1972). Short-term memory in the deaf: A test for speech coding. British Journal of Psychology, 63, 173-180.

Conrad, R. (1979). The deaf schoolchild. London: Harper \& Row.

CRAIK, F. (1970). The fate of primary items in free recall. Journal of Verbal Leaming \& Verbal Behavior, 9, 143-148.

Craine, M. (1977). Short-term memory of the deaf. Hearing Rehabilitation Quarterly, 2, 4-8.

Crowder, R. G. (1976). Principles of learning and memory. Hillsdale, NJ: Earlbaum.

Crowder, R. G. , Morton, J. (1969). Precategorical acoustic storage (PAS). Perception \& Psychophysics, 5, 365-373.

Fromkin, V. A. (Ed.) (1980). Errors in linguistic performance: Slips of the tongue, ear, pen, and hand. New York: Academic Press.

Hakoda, Y., \& Nakamizo, S. (1975). Dual-encoding hypothesis in short-term memory: How does the nature of interpolated task affect information loss? Japanese Psychological Research, 17, 91-97. 
Hanson, V. L. (1982). Short-term recall by deaf signers of American Sign Language: Implications of encoding strategy for order recall. Joumal of Experimental Psychology: Leaming. Memory, \& Cognition, 8, 572-583.

Hanson, V. L., Liberman, I. Y., \& Shankweiler, D. (1984). Linguistic coding by deaf children in relation to beginning reading success. Journal of Experimental Child Psychology, 37, 378-393.

HANSON, V. L., \& LiChTENSTEIN, E. (1986). Short-term memory for printed English words by congenitally deaf signers: Evidence of signbased encoding reconsidered (Status Report on Speech Research No. SR-85). New Haven, CT: Haskins Laboratories.

HEYER, K., \& BARRETT, B. (1971). Selective loss of visual and verbal information in STM by means of visual and verbal interpolated tasks. Psychonomic Science, 25, 100-102.

Hintzman, D. L. (1967). Articulatory coding in short-term memory. Joumal of Verbal Leaming \& Verbal Behavior, 6, 316-321.

Hirsh-Pasex, K., \& Treiman, R. (1982). Recoding in silent reading: Can the deaf child translate print into a more manageable form. Volta Review, 84, 72-82.

Hurley, G., \& KamiL, M. L. (1976). Confusions in memory for tactile presentations of letters. Bulletin of the Psychonomic Society, 8 , 76-78.

Janata, J. W., Joelson, J. M., Joss, K. A., a Herrmann, D. J. (1978). The role of visual and acoustic coding in retrieval from very short-term memory. Bulletin of the Psychonomic Society, 11, 185-187.

JohNSON, D. (1976). Communication characteristics of a young deaf adult population: Techniques for evaluating their communication skills. American Annals of the Deaf, 121, 409-424.

Krakow, R. A., \& HANSON, V. L. (1985). Deaf signers and serial recall in the visual modality: Memory for signs, fingerspelling, and print. Memory \& Cognition, 13, 265-272.

LegGe, D., \& KaminsKa, Z. M. (1975). Memory confusions and memory codes: A simulation of the acoustical similarity effect. Quarterly Joumal of Experimental Psychology, 27, 343-356.

LeVy, B. A., \& CraIK, F. L. (1975). The co-ordination of codes in short-term retention. Quarierly Joumal of Experimental Psychology, 27, 33-45.

Lichtenstein, E. (1985). Deaf working memory processes and English language skills. In D. S. Martin (Ed.), Cognition, education, and deafness: Directions for research and instruction (pp. 143-181). Washington, DC: Gallaudet College Press.

LOCKE, J. L., LocKE, V. L. (1971). Deaf children's phonetic, visual, and dactylic coding in a grapheme recall task. Journal of Experimental Psychology, 89, 142-146.

NeIsser, U. (1967). Cognitive psychology. New York: AppletonCentury-Crofts.

Newkirk, D., Klima, E., Pederson, C., \& Bellugi, U. (1980). Linguistic evidence from slips of the hand. In V. Fromkin (Ed.), Errors in linguistic performance: Slips of the tongue, ear, pen, and hand (pp. 165-197). New York: Academic Press.
Shand, M. (1982). Sign-based short-term coding of American Sign Language signs and printed English words by congenitally deaf signers. Cognitive Psychology, 14, 1-12.

Shulman, H. G. (1970). Encoding and retention of semantic and phonemic information in short-term memory. Journal of Verbal Learning \& Verbal Behavior, 9, 499-508.

Shulman, H. G. (1971). Similarity effects in short-term memory. Psychological Bulletin, 75, 399-415.

Shulman, H. G. (1972). Semantic confusion errors in short-term memory. Journal of Verbal Leaming \& Verbal Behavior, 11, 221-227.

SPERLING, G. (1960). The information available in brief visual presentations. Psychological Monographs, 74(22, Whole No. 498).

SPERLING, G. (1963). A model for visual memory tasks. Human Factors, $5,19-31$.

Sperling, G., Speelman, R. (1970). Acoustic similarity and auditory STM. In D. Norman (Ed.), Models of memory (pp. 151-202). New York: Academic Press.

STOKOE, W. (1960). Sign language structure: An outline of the visual communication system of the American deaf. Studies in Linguistics (Occasional Papers, No. 8).

Wallace, G., \& Corballis, M. C. (1973). Short-term memory and coding strategies in the deaf. Joumal of Experimental Psychology, 99, 334-348.

Wetherick, N. E. (1975). The role of semantic information in shortterm memory. Journal of Verbal Learning \& Verbal Behavior, 14, $471-480$.

Wickens, D. D. (1972). Characteristics of word encoding. In A. Melton \& E. Martin (Eds.), Coding processes in human memory (pp. 194215). New York: Holt, Rinehart, \& Winston.

Zhang, G., \& Simon, H. A. (1985). STM capacity for Chinese words and idioms: Chunking and acoustical loop hypotheses. Memory \& Cognition, 13, 193-201.

\section{APPENDIX}

Stimulus Items Comprising Experimental Lists

\begin{tabular}{ll}
\hline Shoe & Candy \\
Through & Onion \\
New & Apple \\
Show & Japanese \\
No & Jealous \\
Sew & Sour \\
Three & Bored \\
See & Chinese \\
\hline
\end{tabular}

(Manuscript received January 8, 1988; revision accepted for publication November $7,1988$. ) 